\title{
COMMUNICATION AND COMPLIANCE IN EYE CASUALTY
}

\author{
BETH EDMUNDS, PETER J. FRANCIS and ANDREW R. ELKINGTON \\ Southampton
}

\begin{abstract}
SUMMARY
Purpose: This study aimed to assess patients' perceptions and priorities when consulting doctors in eye casualty, to assess their satisfaction with eye casualty and to evaluate and improve patients' level of knowledge and understanding of their treatment.

Methods: A selected consecutive group of 130 patients presenting to eye casualty between 1 July and 15 September 1995 was interviewed by two of the authors prior to collecting their medication. A further group was interviewed again after collecting their medication from the hospital pharmacist. The hospital pharmacist reiterated treatment details when patients collected their medication. Interviews were conducted by means of a questionnaire. There was no inter- or intraobserver variation. Patients' priorities and perceptions were measured as percentages of the group. Patient satisfaction was measured both by a score on a standardised questionnaire and on a visual analogue scale. Patient recall of treatment details was scored as correct or incorrect. The score prior to and after seeing the pharmacist was compared in those patients who were interviewed after collecting their medication.

Results: Among the patients $30.8 \%$ considered themselves emergencies, $20.8 \%$ were referred and the remainder attended for non-urgent reasons. Eightythree per cent $(83.0 \%)$ were wholly satisfied with the consultation. The consultation scored an average of 8.3, SD 1.6, measured on a visual analogue scale of 0-10. When asked the most important aspects of the consultation $54.6 \%$ cited treatment, $25.4 \%$ reassurance and $20.0 \%$ diagnosis. Ninety-six per cent felt that their treatment had been adequately explained; however, $78.3 \%$ made errors when reporting their regimen. A significant improvement in patients' level of recall was found after they had received further information from the hospital pharmacist.

Conclusions: Firstly, this study shows patients' perceptions and priorities when visiting eye casualty. Sec-

From: Southampton Eye Unit, Southampton, UK.

Correspondence to: Beth Edmunds, Royal College of Ophthalmologists, 17 Cornwall Terrace, Regent's Park, London NW14QW, UK.
\end{abstract}

ondly, it demonstrates that patients are generally satisfied with their eye casualty attendance. Thirdly, many patients depart with poor understanding of their eye treatment regime which is likely to affect compliance. Communication between doctors and patients was enhanced by involvement of the hospital pharmacist. This strategy is applicable not only to an ophthalmic casualty unit but also to a wider range of settings and could provide a service standard for future audit.

An important part of many eye units is the provision of an emergency service, which may take many forms but allows patients, both casualties and others, open access to specialist ophthalmic care.

Several studies have detailed the diagnoses and demographics pertaining to attenders. ${ }^{1-6}$ The most common diagnostic groups are trauma, infection and inflammation. The duration of symptoms prior to presentation varies, with the majority of patients attending within a week of symptom onset.

Recently, work undertaken mainly in general practice, has challenged the traditional concept of the doctor-patient interaction. It has become clear that patients hold elaborate and often sophisticated theories of their own illnesses. ${ }^{7}$ They anticipate explanations, often to basic questions, such as why a condition has affected them and why now. Furthermore they expect information and emotional support. It is not clear whether, given the current highly pressured eye casualty set-up, some or any of these expectations are being met.

Compliance with medication prescribed remains a significant problem in clinical practice. Many studies have attempted to identify factors leading to poor compliance..$^{8-15}$ Discriminatory variables include patient knowledge of the medication and the complexity of the regime, technical difficulty in complying, anxiety, and the age and experience of the prescribing doctor. ${ }^{16}$

Given that consultation time is short and the transfer of information beween doctor and patient is 
necessarily concentrated, it is reasonable to suspect that patient compliance is likely to be affected. ${ }^{16-19}$

In the study presented here patients' priorities and satisfaction from eye casualty attendance are examined. The information retained by patients following their consultation is also presented, since this is likely to affect their compliance. The hospital pharmacist was then enrolled in an attempt to improve communication, and patients were re-interviewed after they had received their prescribed medication.

\section{METHODS}

Ethics committee approval was granted prior to the start of the project. One hundred and thirty consecutive patients attending the eye casualty in Southampton Eye Hospital between 1 July and 15 September 1995 were interviewed using a questionnaire following their casualty consultation with either an extended-role nurse or doctor, prior to collection of their medication. The questionnaire employed questions validated by other related studies. ${ }^{12}$ There were no exclusion criteria. Verbal consent was obtained from all participants. Each interview took between 2 and 3 minutes. Information was gathered before comparison with clinical notes so that the interviewers were unaware of the treatment prescribed. When the patient was accompanied, questions were answered as a combined effort by both patient and helper.

Thirty-one randomly selected patients were interviewed again after they had received their medication from the hospital pharmacist, to establish whether they had become better informed. The pharmacist had been instructed specifically to reiterate the name, nature, frequency, duration and route of drug administration.

Interviews were conducted by two of the authors (P.J.F. and B.E.) who are junior ophthalmologists at Southampton Eye Unit. To establish that there was no significant inter-observer variation, a sample of patients were randomly interviewed by one observer followed by the other. There was a concordance of $100 \%$ in answers given.

All information was gathered confidentially. Although the casualty officers and nursing staff were broadly aware of a study being performed, its specific nature was not disclosed in order not to bias outcome.

The chi-squared test was used to analyse the results.

\section{RESULTS}

One hundred and thirty patients visiting the Southampton Eye Casualty were interviewed using a standard questionnaire. Patients' ages ranged from 1 to 90 years (mean 48.5 years). There were 62 males $(47.7 \%)$ and 68 females (52.3\%). Their ocular

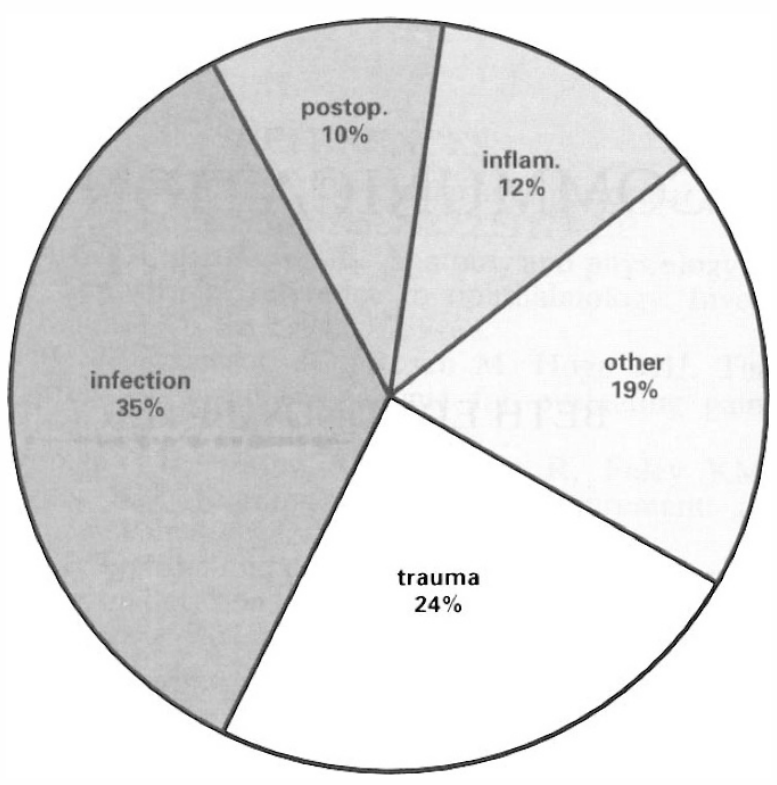

Fig. 1. Diagnoses of patients presenting to eye casualty. Postop, postoperative complications; inflam., inflammation.

conditions are detailed in Fig. 1. For 80 (62\%) patients this was their first visit, but 21 (16.3\%) were making a follow-up visit and 28 (21.7\%) had attended on previous occasions. Fifty-two patients (40.3\%) were examined by a doctor only, 19 (14.7\%) by a nurse only and $58(45 \%)$ by both a doctor and a nurse. There was no significant difference in the responses given between these groups. Twenty-one $(16.4 \%)$ patients were given a leaflet in conjunction with the consultation to explain the nature and treatment of their condition. Fifty-five (45.1\%) patients were accompanied by a spouse, parent, relative, colleague or friend, who was included in the interview as they had been party to the consultation.

Forty $(30.8 \%)$ patients considered themselves emergencies, whilst $27(20.8 \%)$ had been referred by their optician, GP, practice nurse, pharmacist or work nurse. The remaining patients presented for a variety of reasons ranging from 'condition not getting better' or 'getting worse' to simply wanting an 'eye
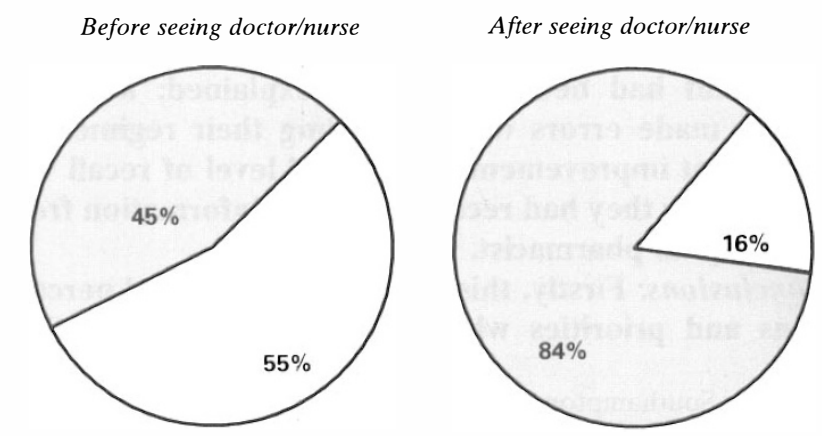

Fig. 2. Proportions of patients knowing their correct diagnosis (grey) or not knowing their correct diagnosis (white) before and after their consultation. 
check'. Twenty patients (18.5\%) would have preferred to visit their GP.

Most patients $(108,83 \%)$ were wholly satisfied with the consultation. The consultation scored an average of 8.3 , SD 1.6, measured on a visual analogue scale of 0 (very poor) to 10 (extremely good).

When asked to list different aspects of the consultation in order of importance, 71 patients $(54.6 \%)$ cited treatment, $33(25.4 \%)$ reassurance and $26(20.0 \%)$ diagnosis as the most important.

Despite 125 (96\%) of patients feeling that their treatment regimen had been adequately explained, $101(78.3 \%)$ made some error in reporting their regime, other than the drug name. Thirteen $(10 \%)$ made errors in the number of treatment items, 115 $(88.4 \%)$ did not know the name of the drug prescribed, $57(44.2 \%)$ did not understand the nature of the drug prescribed, $70(53.5 \%)$ made errors in the prescribed frequency of administration, 60 (46.0\%) did not know the prescribed length of treatment and $19(14.7 \%)$ were uncertain as to which eye or whether both eyes required treatment. One hundred and twenty-one $(93.1 \%)$ patients remembered their follow-up arrangements correctly. Fig. 2 shows the number of patients knowing their correct diagnosis before and after the consultation.

Thirty-one $(23.8 \%)$ patients were interviewed again after receiving their medication and a second explanation of their treatment regimen from the pharmacist. The group did not differ significantly from the whole: the average age was 50.1 years (range 5-76 years) and there were 14 (46\%) males, $17(54 \%)$ females. There was, however, a significant improvement in the reporting of the treatment details after this second interaction. Although 96 (74\%) patients still did not know the name of the drug, $101(77.6 \%)$, compared with $44.2 \%(p<0.01)$, were now aware of its nature. Only $17(12.8 \%)$ patients, compared with $53.5 \%(p<0.01)$, made errors in the prescribed frequency of administration; 21 $(16.1 \%)$, compared with $46.0 \% \quad(p<0.01)$, did not know the prescribed length of treatment; and 8 (6.5\%), compared with $14.7 \%(p<0.2)$ were uncertain as to which eye or whether both eyes required treatment. No patients made errors in the number of treatment items prescribed. All patients felt that, having seen the pharmacist, their treatment regimen had been fully explained to them.

\section{DISCUSSION}

This study set out firstly to assess patient satisfaction with eye casualty attendance and secondly to assess the adequacy of doctors' communication with patients about their treatment regime. A further group of patients was questioned after seeing the hospital pharmacist, with a marked improvement in their recall of the details of treatment.

Patients were interviewed by two of the authors using a questionnaire prepared with the advice of the University Department of Clinical Psychology. The questions concerning patient satisfaction have previously been validated. ${ }^{10}$ There was no interobserver variation, with $100 \%$ concordance between the two interviewers. The questionnaire was easy to use and the study was generally welcomed by participating patients.

Whilst it is reasonable to surmise that patients attend the eye casualty because they want help with their illnesses, it is not clear whether the service provided adequately meets their expectations. Brackenbury ${ }^{20}$ wrote in 1935 of the doctor-patient interaction that patients wanted clinical competence; unable to judge this for themselves, they had to rely on the professional integrity of doctors. Furthermore, Cassidy ${ }^{21}$ suggested that doctors only sought from the patient 'an account of their symptoms, as concise as possible and chronological', with the patient being regarded as rather passive. In essence, patients might know what they wanted, but doctors knew what patients needed. Recently, this traditional concept has been challenged and it is now believed that patients have more sophisticated expectations. It is gratifying to note, therefore, that patients reported a high degree of satisfaction with the consultation. Eighty-three per cent were wholly satisfied and gave an overall rating of 8.3 , SD 1.6, on a visual analogue scale of 0 to 10 . A satisfactory doctor-patient interaction is important to maximise communication and transfer of information. Patients felt at ease with the doctor, had enough time and felt that the doctor listened. They also indicated that an opportunity had been given for questions, frustrations, emotions and worries to be voiced, and were satisfied with the explanation of the diagnosis and treatment.

However, $78.3 \%$ of patients made some error (disregarding the name of the drug) in remembering their treatment. This is surprising as $96 \%$ felt that their treatment regimen had been adequately explained and $54.6 \%$ had rated the treatment as the most important aspect of the consultation. Patients were asked to recall the name, nature, route, frequency and length of administration of the medication prescribed. Whilst it is of less importance that the patient should remember the actual name of the drug, remembering the frequency and length of treatment is paramount to a satisfactory outcome. An understanding of the nature of the treatment is also important as it has been shown that knowledge of the drug improves compliance. ${ }^{17}$

Those patients who were questioned after seeing the pharmacist showed a significant improvement in their recollection of treatment details. Whilst it is 
possible that reinforcement by another health care professional may be equally effective, the authors feel that engaging the help of pharmacy staff is the most efficient and convenient method available.

Patient compliance is adversely affected by young practitioners with few years' experience. ${ }^{17}$ Casualty officers usually fall into this category and are often too busy to spend time explaining treatment details when so much other information has to be exchanged. Information leaflets were supplied to $21 \%$ of patients but cannot be expected to be flexible enough to accommodate individual treatment schedules. The pharmacist is ideally placed to perform this function. This could release the casualty officer to spend more time discussing the diagnosis and reassuring the patient where appropriate. Almost $50 \%$ of patients felt that diagnosis and reassurance were the most important aspects of the consultation.

\section{CONCLUSION AND RECOMMENDATION}

This study demonstrates a strategy to improve communication between health professionals and patients. Furthermore this improved communication is in an area vital to the success of treatment and compliance. Communication between doctors and patients in a casualty setting was enhanced by involvement of the pharmacist. This second interaction with patients resulted in a statistically significant improvement in patients' recall of treatment details. This strategy is applicable in a wider range of settings and is not unique to an ophthalmic casualty unit. It is relevant to patients attending general as well as specialist casualty units and outpatient clinics where consultations are often similarly concentrated.

The authors suggest that the results of this study be used as a service standard for further audit of eye casualty services.

The authors would like to extend their thanks to the pharmacy staff of Southampton Eye Unit (Sue Wakelin and Su Moughtin) who helped in the conduct of this study.

Key words: Communication, Compliance, Pharmacist, Accident \& Emergency, Patient satisfaction.

\section{REFERENCES}

1. Jones NP, Hayward JM, Khaw PT, Elkington AR. Function of an ophthalmic A\&E department: results of six month survey. BMJ 1986;292:188-90.

2. Chiapella AP, Rosenthal AR. One year in an eye casualty clinic. Br J Ophthalmol 1985;69:865-70.

3. Edwards RS. Ophthalmic emergencies in a district general hospital casualty department. Br J Ophthalmol 1987;71:938-42.

4. Verma L, Arora R, Tewari HK, Khosla PK. Eye casualty department. J R Soc Med 1994;87:217-8.

5. George S, Read S, Westlake L, Williams B, FraserMoodie A, Pritty P. Evaluation of nurse triage in a British A\&E department. BMJ 1992;304:876-8.

6. Sheldrick JH, Vernon SA, Wilson A, Read SJ. Demand incidence of ophthalmic disease in a defined urban population. BMJ 1992;305:904-5.

7. Armstrong D. What do patients want? BMJ 1991; 303:261-2.

8. Martin E, Russell D, Goodwin S, Chapman R, North M, Sheridan P. Why patients consult and what happens when they do. BMJ 1989;303:261-2.

9. Jones CS, McGowan A. Self referral to an A\&E department for another opinion. BMJ 1989;298:1182-3.

10. Rashid A, Forman W, Jagger C, Mann R. Consultations in general practice: a comparison of patients' and doctors" satisfaction. BMJ 1989;299:1015-6.

11. Virji A. A study of patients attending without appointments in an urban general practice. BMJ 1990;301:22-6.

12. Morrell DC, Evans ME, Morris RW, Roland MO. The five minute consultation: effect of time on clinical content and patient satisfaction. BMJ 1986;292:870-3.

13. Singh S. Self referral to A\&E: patients' perspectives. BMJ 1988;297:1179-80.

14. Appelbaum PS, Grisso T. Assessing patients' capacities to consent to treatment. N Engl J Med 1988;319: $1635-8$.

15. Williams GH, Wood PH. Common sense beliefs about illness: a mediating role for the doctor. Lancet 1986;II:1435-7.

16. Buckalew LW, Sallis RE. Patient compliance and medication prescription. J Clin Psychol 1986;42:49-53.

17. Cockburn J, Gibberd R, Reid A, Sanson-Fisher R. Determinants of non-compliance with short term antibiotic regimens. BMJ (Clin Res Ed) 1987; 295:814-8.

18. Herrier RN, Boyce RW. Does counselling improve compliance? Am Pharmacy 1995;35:11-2.

19. Williford SL, Johnson DF. Impact of pharmacist counselling on medication knowledge and compliance. Military Med 1995;160:561-4.

20. Brackenbury HB. Patient and doctor. London: Hodder and Stoughton, 1935.

21. Cassidy M. Doctor and patient. Lancet 1938;I:175-9. 